\title{
Rotations in Minkowski spacetime
}

\section{I.Guerrero-Moreno, G. Leija-Hernández, J. López-Bonilla}

ESIME-Zacatenco, IPN, Edif. 5, 1er. Piso, Col. Lindavista CP 07738, México DF;

jlopezb@ipn.mx

Keywords: Lorentz matrix, Null tetrad of Newman-Penrose

Abstract. With the relation of Olinde Rodrigues-Cartan is obtained an expression for the Lorentz matrix, and it is transformed to a better form for the Newman-Penrose formalism, thus it is possible to realize rotations of the null tetrad of NP.

\section{Introduction}

Here we employ the notation and conventions of [1]. The Olinde Rodrigues [2]-Cartan [3] expression:

$$
\left(\begin{array}{cc}
\tilde{x}^{0}+\tilde{x}^{3} & \tilde{x}^{1}-i \tilde{x}^{2} \\
\tilde{x}^{1}+i \tilde{x}^{2} & \tilde{x}^{0}-\tilde{x}^{3}
\end{array}\right)=\left(\begin{array}{cc}
\alpha & \beta \\
\gamma & \delta
\end{array}\right)\left(\begin{array}{cc}
x^{0}+x^{3} & x^{1}-i x^{2} \\
x^{1}+i x^{2} & x^{0}-x^{3}
\end{array}\right)\left(\begin{array}{cc}
\bar{\alpha} & \bar{\gamma} \\
\bar{\beta} & \bar{\delta}
\end{array}\right),
$$

where $\alpha, \beta, \gamma, \delta$ are arbitrary complex numbers verifying the condition $\alpha \delta-\beta \gamma=1$, implies six degrees of freedom for the Lorentz matrix $L=\left(L^{v}{ }_{\mu}\right)$ between the frames of reference $\left(x^{\mu}\right)=(c t, x, y, z)$ and $\left(\tilde{x}^{v}\right)$ :

$$
\tilde{x}^{v}=L_{\mu}^{v} x^{\mu} .
$$

From (1) and (2) we obtain the relations [4-8]:

$$
\begin{array}{lll}
L^{0}{ }_{0}=\frac{1}{2}(\alpha \bar{\alpha}+\beta \bar{\beta}+\gamma \bar{\gamma}+\delta \bar{\delta}), & L^{1}{ }_{0}=\frac{1}{2}(\bar{\alpha} \gamma+\bar{\beta} \delta)+c c, & L^{2}{ }_{0}=\frac{i}{2}(\alpha \bar{\gamma}-\bar{\beta} \delta)+c c, \\
L^{0}{ }_{1}=\frac{1}{2}(\bar{\alpha} \beta+\bar{\gamma} \delta)+c c, & L^{1}{ }_{1}=\frac{1}{2}(\bar{\alpha} \delta+\beta \bar{\gamma})+c c, & L^{2}{ }_{1}=\frac{i}{2}(\alpha \bar{\delta}+\beta \bar{\gamma})+c c, \\
L^{0}{ }_{2}=\frac{i}{2}(\bar{\alpha} \beta+\bar{\gamma} \delta)+c c, & L^{1}{ }_{2}=\frac{i}{2}(\bar{\alpha} \delta+\beta \bar{\gamma})+c c, & L^{2}{ }_{2}=\frac{1}{2}(\bar{\alpha} \delta-\bar{\beta} \gamma)+c c, \quad(3) \\
L^{0}{ }_{3}=\frac{1}{2}(\alpha \bar{\alpha}-\beta \bar{\beta}+\gamma \bar{\gamma}-\delta \bar{\delta}), & L^{1}{ }_{3}=\frac{1}{2}(\bar{\alpha} \gamma-\bar{\beta} \delta)+c c, & L^{2}{ }_{3}=\frac{i}{2}(\alpha \bar{\gamma}+\bar{\beta} \delta)+c c, \\
L^{3}{ }_{0}=\frac{1}{2}(\alpha \bar{\alpha}+\beta \bar{\beta}-\gamma \bar{\gamma}-\delta \bar{\delta}), & L^{3}{ }_{1}=\frac{1}{2}(\bar{\alpha} \beta-\bar{\gamma} \delta)+c c, & L^{3}{ }_{2}=\frac{i}{2}(\bar{\alpha} \beta-\bar{\gamma} \delta)+c c, \\
L^{3}{ }_{3}=\frac{1}{2}(\alpha \bar{\alpha}-\beta \bar{\beta}-\gamma \bar{\gamma}+\delta \bar{\delta}), & \alpha \delta-\beta \gamma=1, &
\end{array}
$$

where cc means the complex conjugate of all the previous terms.

In Sec. 2, into L we eliminate $\alpha, \beta, \gamma, \delta$ in favour of another quantities better adapted to the null tetrads of Newman-Penrose (NP) [9-11], thus from (3) we shall obtain the expressions deduced in [12-14].

\section{Rotations in special relativity}

If in (3) we realize the changes $\left[\tau=\frac{1}{\sqrt{1-\mathrm{r} \Omega}}\right]$ :

$\alpha=-\tau \exp \left(-\frac{A+i B}{2}\right), \quad \beta=\tau \exp \left(-\frac{A+i B}{2}\right) \bar{\Gamma}, \quad \gamma=\tau \exp \left(\frac{A+i B}{2}\right) \bar{\Omega}, \quad \delta=-\tau \exp \left(\frac{A+i B}{2}\right)$, 
where A, B are real and $\Gamma, \Omega$ are complex with $\Gamma \Omega \neq 1$ (it is simple to verify that (4) respects the requirement $\alpha \delta-\beta \gamma=1)$, we deduce the relations of $[12-14]^{\left[Q=\frac{1}{2|1-\mathrm{r} \Omega|}\right] \text { : }}$

$$
\begin{aligned}
& L^{0}{ }_{0}=\mathrm{Q}\left[e^{\mathrm{A}}(1+\Omega \bar{\Omega})+e^{-\mathrm{A}}(1+\Gamma \bar{\Gamma})\right], \quad L^{1}{ }_{0}=-\mathrm{Q}\left[e^{i \mathrm{~B}}(\Gamma+\bar{\Omega})+c c\right], \quad L^{2}{ }_{0}=-i \mathrm{Q}\left[e^{-i \mathrm{~B}}(\Omega+\right. \\
& \bar{\Gamma})-c c],
\end{aligned}
$$

$L^{0}{ }_{1}=-\mathrm{Q}\left[e^{\mathrm{A}}(\Omega+\bar{\Omega})+e^{-\mathrm{A}}(\Gamma+\bar{\Gamma})\right], \quad L^{1}{ }_{1}=\mathrm{Q}\left[e^{i \mathrm{~B}}(1+\bar{\Omega} \Gamma)+c c\right], \quad L^{2}{ }_{1}=i \mathrm{Q}\left[e^{-i \mathrm{~B}}(1+\right.$ $\Omega \bar{\Gamma})-c c]$,

$L^{0}{ }_{2}=i \mathrm{Q}\left[e^{\mathrm{A}}(\bar{\Omega}-\Omega)+e^{-\mathrm{A}}(\Gamma-\bar{\Gamma})\right], \quad L^{1}{ }_{2}=i \mathrm{Q}\left[e^{i \mathrm{~B}}(1-\bar{\Omega} \Gamma)-c c\right], \quad L^{2}{ }_{2}=-\mathrm{Q}\left[e^{-i \mathrm{~B}}(\Omega \bar{\Gamma}-\right.$ 1) $+c c]$,

$L^{0}{ }_{3}=\mathrm{Q}\left[e^{\mathrm{A}}(\Omega \bar{\Omega}-1)+e^{-\mathrm{A}}(1-\Gamma \bar{\Gamma})\right], \quad L^{1}{ }_{3}=\mathrm{Q}\left[e^{i \mathrm{~B}}(\Gamma-\bar{\Omega})+c c\right], \quad L^{2}{ }_{3}=i \mathrm{Q}\left[e^{-i \mathrm{~B}}(\bar{\Gamma}-\Omega)-\right.$ $c c]$,

$$
\begin{aligned}
& L^{3}{ }_{0}=\mathrm{Q}\left[-e^{\mathrm{A}}(1+\Omega \bar{\Omega})+e^{-\mathrm{A}}(1+\Gamma \bar{\Gamma})\right], L^{3}{ }_{1}=\mathrm{Q}\left[e^{\mathrm{A}}(\Omega+\bar{\Omega})-e^{-\mathrm{A}}(\Gamma+\bar{\Gamma})\right], \\
& L^{3}{ }_{2}=i \mathrm{Q}\left[e^{\mathbf{A}}(\Omega-\bar{\Omega})+e^{-\mathrm{A}}(\Gamma-\bar{\Gamma})\right], \quad L^{3}{ }_{3}=\mathrm{Q}\left[e^{\mathbf{A}}(1-\Omega \bar{\Omega})+e^{-\mathrm{A}}(1-\Gamma \bar{\Gamma})\right] .
\end{aligned}
$$

In [15] we showed that an orthonormal real tetrad experiments a rotation in Minkowski space under a Lorentz matrix:

$$
\tilde{e}_{\mu}^{(a)}=L^{a}{ }_{b} e^{(b)}{ }_{\mu},
$$

such that $e_{\mu}^{(0)}=e_{(0) \mu}$ and $e^{(j)}{ }_{v}=-e_{(j) v}, j=1,2,3$, this due to the metric tensor Diag (1,-1,$1,-1)$. From (5), (6) and the definitions [9-11]:

$l^{v}=\frac{1}{\sqrt{2}}\left(e_{(0)}^{v}+e_{(3)}^{v}\right), n^{v}=\frac{1}{\sqrt{2}}\left(e_{(0)}^{v}-e_{(3)}^{v}\right), m^{v}=\frac{1}{\sqrt{2}}\left(e_{(1)}^{v}-i e_{(2)}{ }^{v}\right), \bar{m}^{v}=\frac{1}{\sqrt{2}}\left(e_{(1)}^{v}+\right.$ $\left.i e_{(2)} v\right)$,

it is possible to calculate the rotation of this null tetrad of NP $\left[\mathrm{C}=\frac{1}{\mid 1-\mathrm{r} \Omega}\right]$ :

$$
\begin{array}{ll}
\tilde{l}^{\mu}=\mathrm{C} e^{\mathrm{A}}\left(l^{\mu}+\Omega \bar{\Omega} n^{\mu}+\bar{\Omega} m^{\mu}+\Omega \bar{m}^{\mu}\right), & \tilde{n}^{\mu}=\mathrm{C} e^{-\mathrm{A}}\left(n^{\mu}+\Gamma \bar{\Gamma} l^{\mu}+\Gamma m^{\mu}+\bar{\Gamma} \bar{m}^{\mu}\right), \\
\tilde{m}^{\mu}=\mathrm{C} e^{-i \mathrm{~B}}\left(\bar{\Gamma} l^{\mu}+\Omega n^{\mu}+m^{\mu}+\bar{\Gamma} \Omega \bar{m}^{\mu}\right), & \widetilde{m}^{\mu}=\mathrm{C} e^{i \mathrm{~B}}\left(\Gamma l^{\mu}+\bar{\Omega} n^{\mu}+\bar{m}^{\mu}+\Gamma \bar{\Omega} m^{\mu}\right),
\end{array}
$$

which is very useful for several applications in relativity [9-11, 16, 17]; in (8) it is easy to check that $\tilde{l}^{\mu} \tilde{n}_{\mu}=-\tilde{m}^{\mu} \widetilde{\bar{m}}_{\mu}=1$.

Thus, in the literature it is natural to employ three types of rotations:

Class I: $\quad \Omega=0 . \quad l^{\mu}$ preserves its direction.

$\tilde{l}^{\mu}=e^{\mathrm{A}} l^{\mu}, \tilde{n}^{\mu}=e^{-\mathrm{A}}\left(n^{\mu}+\Gamma \bar{\Gamma} l^{\mu}+\Gamma m^{\mu}+\bar{\Gamma} \bar{m}^{\mu}\right), \quad \tilde{m}^{\mu}=e^{-i \mathrm{~B}}\left(\bar{\Gamma} l^{\mu}+m^{\mu}\right), \tilde{\bar{m}}^{\mu}=$ $e^{i \mathrm{~B}}\left(\Gamma l^{\mu}+\bar{m}^{\mu}\right)$,

Class II: $\quad \Gamma=0 . \quad n^{v}$ maintains its direction. 


$$
\begin{aligned}
& \tilde{l}^{v}=e^{\mathrm{A}}\left(l^{v}+\Omega \bar{\Omega} n^{v}+\bar{\Omega} m^{v}+\Omega \bar{m}^{v}\right), \tilde{n}^{v}=e^{-\mathrm{A}} n^{v}, \tilde{m}^{v}=e^{-i \mathrm{~B}}\left(\Omega n^{v}+m^{v}\right), \widetilde{\bar{m}}^{v}= \\
& e^{i \mathrm{~B}}\left(\bar{\Omega} n^{v}+\bar{m}^{v}\right),
\end{aligned}
$$

Class III: $\Omega=\Gamma=0$.

$$
\tilde{l}^{\mu}=e^{\mathrm{A}} l^{\mu}, \quad \tilde{n}^{\mu}=e^{-\mathrm{A}} n^{\mu}, \quad \tilde{m}^{\mu}=e^{-i \mathrm{~B}} m^{\mu}, \quad \tilde{\bar{m}}^{\mu}=e^{i \mathrm{~B}} \bar{m}^{\mu},
$$

which permit to determine the evolution of, for example, the spin coefficients, the NP components of Weyl, Ricci and Lanczos tensors, etc., under a rotation of the null tetrad. This is important because it is usual to make rotations to align $l^{\mu}$ or/and $n^{\mu}$ with the principal directions of Cartan [18]-Debever [19]-Penrose [20] for the conformal tensor or the Faraday's electromagnetic tensor [21], which gives great simplification in many relativistic calculations.

\section{References}

[1]. A. Hernández G., R. López-Vázquez, J. Rivera R., J. López-Bonilla, Faraday tensor and Maxwell spinor, Journal de Ciencia e Ingeniería 6, No. 1 (2014)

[2]. B. Olinde Rodrigues, Des lois géométriques qui régissent les déplacements d'un systéme solide, Journal de Math. (Liouville) 5 (1840) 380-440

[3]. E. Cartan, Les groupes projectifs qui ne laissent invariante aucune multiplicité plane, Bull. Soc. Math. de France 41 (1913) 53-96

[4]. Ju. Rumer, Spinorial analysis, Moscow (1936)

[5]. J. Aharoni, The special theory of relativity, Clarendon Press, Oxford (1959)

[6]. J. L. Synge, Relativity: the special theory, North-Holland, Amsterdam (1965)

[7]. J. López-Bonilla, J. Morales, G. Ovando, On the homogeneous Lorentz transformation, Bull. Allahabad Math. Soc. 17 (2002) 53-58

[8]. M. Acevedo M., J. López-Bonilla, M. Sánchez M., Quaternions, Maxwell equations and Lorentz transformations, Apeiron 12, No. 4 (2005) 371-384

[9]. E. Newman, R. Penrose, An approach to gravitational radiation by a method of spin coefficients, J. Math. Phys. 3, No. 3 (1962) 566-578

[10]. S. J. Campbell, J. Wainwright, Algebraic computing and the Newman-Penrose formalism in general relativity, Gen. Rel. Grav. 8, No. 12 (1977) 987-1001

[11]. D. Kramer, H. Stephani, M. MacCallum, E. Herlt, Exact solutions of Einstein's field equations, Cambridge University Press (1980)

[12]. R. K. Sachs, Gravitational waves in general relativity VI: The outgoing radiation condition, Proc. Roy. Soc. London A264 (1961) 309-337

[13]. P. J. Greenberg, J. P. Knauer, Stud. Appl. Math. 53 (1974) 165

[14]. M. Carmeli, Group theory and general relativity, McGraw-Hill, New York (1977)

[15]. R. Linares, J. López-Bonilla, J. Quino, Real tetrads and Lorentz transformations, Bol. Soc. Cub. Mat. Comp. 8, No. 2 (2010) 141-153

[16]. P. J. Greenberg, The algebra of the Riemann curvature tensor in general relativity: Preliminaries, Stud. Appl. Math. 51 (1972) 277

[17]. S. Chandrasekhar, An introduction to the theory of the Kerr metric and its perturbations, in General Relativity, Eds. S. W. Hawking, W. Israel, Cambridge University Press (1979) Chap. 7

[18]. E. Cartan, Sur les espaces conformes généralisés et l'Univers optique, C. R. Acad. Sci. Paris 174 (1922) 857-859

[19]. R. Debever, Le rayonnement gravitationnel: Le tenseur de Riemann en relativité générale, Cah. de Phys 168-169 (1964) 303

[20]. R. Penrose, A spinor approach to general relativity, Ann. of Phys. 10 (1960) 171-201

[21]. J. López-Bonilla, R. Meneses, M. Turgut, Faraday tensor: its algebraic structure, J. Vect. Rel. 4, No. 3(2009) 23-32 